\title{
Partial hepatectomy for spontaneous tumor rupture in patients with hepatocellular carcinoma: a retrospective cohort study
}

This article was published in the following Dove Press journal:

Cancer Management and Research

19 October 2017

Number of times this article has been viewed

\author{
Qian Zhu',* \\ Guo-Liang Qiao 2,* \\ Chang $\mathrm{Xu}^{3}$ \\ De-Liang Guo' \\ Jie Tang' \\ Rui Duan' \\ Yun Li'
}

'Department of Hepatobiliary and Pancreatic Surgery, Jingmen First People's Hospital, Jingmen, Hubei Province, ${ }^{2}$ Department of Medical Oncology, Capital Medical University Cancer Center, Beijing Shijitan Hospital, Beijing, ${ }^{3}$ Second Department of Biliary Surgery, Eastern

Hepatobiliary Surgery Hospital, Second Military Medical University, Shanghai, China

*These authors contributed equally to this work
Correspondence: Yun Li Department of Hepatobiliary and Pancreatic Surgery, Jingmen First People's Hospital, 67 Xiangshan Avenue, Jingmen 448000, Hubei Province, China Email ehbhliyun@I63.com
Background: The impact of ruptured hepatocellular carcinoma (HCC) on a patients outcome after hepatic resection remains insufficient. We aimed to identify the independent predictive factors of spontaneous tumor rupture (STR) for curative resection of HCC and to investigate the impact of STR of HCC on long-term survival after resection.

Patients and methods: The clinicopathological parameters of 106 patients with ruptured HCC and 201 patients with non-ruptured HCC who underwent hepatic resection from 2007 to 2011 were investigated. Clinical features and factors associated with the clinical outcomes were compared between both groups.

Results: Of 774 HCC patients who underwent surgical resection, 106 (13.7\%) had tumor rupture. Multivariate stepwise logistic regression analysis revealed hypertension, liver cirrhosis, total bilirubin (TB), tumor size and ascites to be independent prognostic factors for patients with ruptured HCC. The overall survival (OS) of patients in the ruptured HCC group was significantly poorer compared with those in the non-ruptured HCC group. The 1-, 3- and 5 -year OS rates were $77.7 \%, 56.9 \%$ and $41.6 \%$, respectively, in the non-ruptured HCC group and $37.7 \%, 19.7 \%, 14 . \%$, respectively, in the ruptured HCC group $(P<0.001)$. Similar OS rates were found in patients with non-ruptured and ruptured HCC; patients in the non-ruptured HCC group had a significantly better recurrence-free survival (RFS) rate compared with those in the ruptured group $(P=0.016)$.

Conclusion: The presence of hypertension, liver cirrhosis, higher TB levels, tumor size $>5 \mathrm{~cm}$ and ascites are the independent indicators of poorer prognosis for patients undergoing hepatic resection after ruptured HCC. The present study confirmed that tumor rupture itself had a negative impact on patient survival, but hepatic resection, when technically feasible, is safe and appropriate in selected patients and can result in OS and RFS rates comparable to that of patients with non-ruptured HCC.

Keywords: hepatocellular carcinoma, spontaneous rupture, hepatectomy, overall survival, recurrence-free survival

\section{Background}

Hepatocellular carcinoma (HCC) is one of the leading etiologies of global cancerrelated mortalities, which is especially prevalent in China. ${ }^{1-4}$ Spontaneous tumor rupture (STR), which is an uncommon but potentially fatal complication of $\mathrm{HCC}$, accompanied by intraperitoneal hemorrhage is a potentially fatal condition, with reported incidence ranging between $10 \%$ and $15 \% .^{5-8}$

When confronted with this condition, hemostasis should be urgently attempted as the initial treatment, followed by hepatic resection as secondary. Partial hepatectomy 
is a well-validated treatment strategy for $\mathrm{HCC}$, which has become more feasible with satisfactory safety and efficacy profiles due to more advanced surgical technique and perioperative care. Partial hepatectomy is an effective treatment for spontaneously ruptured HCC, through which long-term survival can be achieved in selected patients. ${ }^{9,10}$ It should be performed when technically feasible as either an emergency or a staged operation (following embolization or other hemostatic procedures). However, the short- and long-term outcomes of patients undergoing partial hepatectomy due to spontaneous rupture of HCC warrant further validations. ${ }^{3}$ The present study aimed to investigate the clinicopathological characteristics of ruptured HCC and to demonstrate the impact of tumor rupture and other prognostic factors on the prognosis of this condition in a large patient cohort.

\section{Patients and methods}

This is a retrospective study of consecutive patients who underwent partial hepatectomy for HCC between April 2007 and November 2011 at the Department of Hepatic Surgery, Eastern Hepatobiliary Surgery Hospital, Shanghai, China. Data were prospectively collected in a computer database. Data analysis was done retrospectively. Additional data were obtained by reviewing medical records. The exclusion criteria were 1) incomplete clinical data; 2) presence of severe comorbidities that could affect life expectancy, such as with a history of severe cardiac disorders; 3 ) preoperative portal vein embolization; 4) surgical portosystemic shunts before or at the same time as hepatic resection, were treated as an emergency and 5) a palliative resection or had presented at pathologic examination. According to whether the HCC had ruptured or not, all participants were divided into two groups. Informed consent was obtained from all participants. The research protocol of this study was discussed and approved by the Clinical Research Ethics Committee of Eastern Hepatobiliary Surgery Hospital.

\section{Preoperative care, surgical procedures and follow-up}

Preoperative checkups, including electrocardiography, chest $\mathrm{X}$-ray, complete blood counts, liver and renal function tests, serum $\alpha$-fetoprotein (AFP) level, serological markers for hepatitis B and coagulation profile, were done for every patient. Tumor location and extent were assessed by computed tomography (CT) and/or magnetic resonance imaging (MRI), and resectability was determined accordingly. A previously described criterion was used for resection and remained identical over the study period. ${ }^{11}$ Child-Pugh grade $\mathrm{C}$ liver function was considered as an absolute contraindication to partial hepatectomy. ${ }^{12}$

All operations were performed by experienced surgeons. Pringle manoeuver was routinely used with cycles of clamp/ unclamp for $15 / 5$ minutes. Transection of the hepatic parenchyma was done using the clamp-crushing technique, and hemostasis was done with suture ligations and argon beam coagulators. Anatomical resection was preferred when possible, while nonanatomical resection was reserved for tumors that were peripherally located or situated at the junction of several liver segments, as well as for patients with serious cirrhosis. Major hepatectomy was defined as resection of three or more Couinaud liver segments, while minor hepatectomy was defined as resection of fewer than three segments. The presence of cirrhosis was confirmed by histopathological examination.

When all microscopic and macroscopic tumors were resected, it was defined as $\mathrm{R} 0$ resection. A complication was defined as the occurrence of postoperative pulmonary, renal, cardiac or liver failure; biliary complications; sepsis of any etiology and wound complications. Ascites and pleural effusion that required diuretics or paracentesis were defined as morbidities. Postoperative liver failure was defined by a postoperative serum total bilirubin (TB) level of $>60 \mu \mathrm{mol} / \mathrm{L}$, prothrombin time $>18 \mathrm{~s}$ and/or the development of postoperative hepatic encephalopathy. Postoperative conditions and complications were assessed daily from the day of surgery until discharge.

Patients were followed-up every 2-3 months during the first year after surgery and 3-6 months thereafter until November 30, 2016. All patients were followed up with AFP measurement, CT or MRI and chest X-ray at 4 weeks after operation for 6 months at a 2-month interval and every 3 months thereafter. Patients underwent positron emission tomography, digital subtraction angiography or bone scan when necessary. All follow-up examinations were done by two physicians blinded of patient information and study data. Diagnostic criteria for recurrences were the same as for preoperative diagnosis of HCC. Once recurrence occurred, treatment plan was made based on the pattern of recurrence, hepatic functional reserve and the patient's general condition. A multidisciplinary approach, including re-resection, local ablative therapy, transcatheter arterial chemoembolization (TACE), external irradiation, systemic chemotherapy/ immunotherapy or sorafenib (since 2008), was adopted for patients with recurrence. 


\section{Special procedures for ruptured HCC}

The diagnosis of HCC rupture was based on symptoms and signs upon admission, as well as bedside ultrasonography. For hemodynamically unstable patients, active resuscitation with intravascular fluid was initiated with correction of coagulopathy and patients were monitored closely in the ward or in the intensive care unit. In the majority of patients, bleeding would stop spontaneously with conservative treatment and watchful waiting. When the bleeding exacerbated, emergency transcatheter arterial embolization (TAE) was done. Some of the patients in this study had already undergone TAE before transferring to the authors' hospital for further treatment.

When bleeding could not be controlled by nonsurgical approaches, emergency laparotomy was indicated. For patients with resectable tumor, adequate liver functional reserves and satisfactory general condition, partial hepatectomy was conducted. Surgical hemostatic procedures, such as packing or hepatic artery ligation, suturing, plication and alcohol injection, were used to stop active bleeding at the site of rupture.

Pringle manoeuver and occlusion of the hepatic artery proper were used in emergency partial hepatectomy. ${ }^{13}$ Tumor was reassessed for resectability after clot removal in the peritoneal cavity. Ideally, a resection margin of $>1 \mathrm{~cm}$ was planned. After hepatectomy, peritoneal lavage with distilled water (DWPL; 5000-10000 mL) was done, and 5-fluorouracil (5-FU; $500 \mathrm{mg}$ ) was left in the abdominal cavity before closure. ${ }^{14}$

Staged hepatectomy would be planned 2-6 weeks after the episode of spontaneous rupture for patients with resectable HCC whose bleeding had stopped either spontaneously or with laparotomy.

\section{Statistical analysis}

All data are presented as mean values with standard deviation (SD) or percentages. Nominal data were compared with the Pearson $\chi^{2}$ test; multiple forward stepwise logistic regressions were used when appropriate. The distribution of continuous data was tested using the Kolmogorov-Smirnov test; values are shown as mean (SD) if distributed normally and as median (range) otherwise. These data were analyzed by mean values of the independent samples $t$-test and Wilcoxon rank-sum test (Mann-Whitney $U$-test). Survival was calculated and compared with the log-rank test and presented with the Kaplan-Meier approach. Hospital mortality was defined as death during the hospital stay or within 60 days of surgery. Overall survival (OS) was defined as the length of time between surgery and death or the last follow-up examination.
Recurrence-free survival (RFS) was calculated from the date of tumor resection until tumor recurrence or last observation. To identify factors predictive of survival, univariable and multivariable analyses were performed using the log-rank test and Cox proportional hazards model, respectively. All statistical analyses were conducted using SPSS software version 19.0 (IBM Corporation, Armonk, NY, USA). $P<0.05$ was considered as statistically significant.

\section{Results}

\section{Characteristics of ruptured HCC}

During the study period, a total of 4209 patients with HCC were admitted in the authors' unit, of which 200 (4.8\%) had tumor rupture. Of the 774 patients who underwent elective partial hepatectomy, 106 (13.7\%) had STR before surgery (Figure 1). Patients included in the study were divided into ruptured $(n=106)$ and non-ruptured $(n=201)$ groups. Both groups underwent hepatectomy from the same team of surgeons. In all, 94 patients with ruptured HCC did not receive hepatectomy due to various reasons: preoperative decision of unresectable HCC $(51,54.3 \%)$, intraoperative decision as not suitable for hepatectomy (4, 4.3\%), inadequate liver functional reserve $(17,18.1 \%)$, poor general condition (10, $10.6 \%)$, patient refusal $(3,3.2 \%)$ or for undefined reasons $(9,9.5 \%)$.

A total of 99 men and seven women with a mean age of 47.9 years (range $22-75$ years) were in the ruptured HCC group. No significant difference was found in age distributions and sex ratios between the ruptured and non-ruptured groups (Table 1). Sudden-onset abdominal pain (71 patients, $67 \%$ ) and shock (54 patients, $51 \%$ ) were more common in the ruptured HCC group. However, patients in the ruptured group presented with significantly more symptoms and higher prevalence of cirrhosis than those in the non-ruptured group. Meanwhile, patients in the ruptured group were more likely to have the presence of arterial hypertension (hypertension), ascites, higher Child-Pugh grade, a lower serum albumin level, a higher TB level, a higher serum AFP level, seropositivity for hepatitis B and worse tumor characteristics (including size,).

\section{Perioperative outcomes}

Perioperative parameters, including macroscopic and microscopic findings, are summarized in Table 2. Tumor size in the ruptured HCC group was significantly greater than that in the non-ruptured group $(P=0.002)$. Characteristics of the tumors, such as capsule formation, vascular invasion, satellite lesions, recurrence rate and surgical margins, were similar 


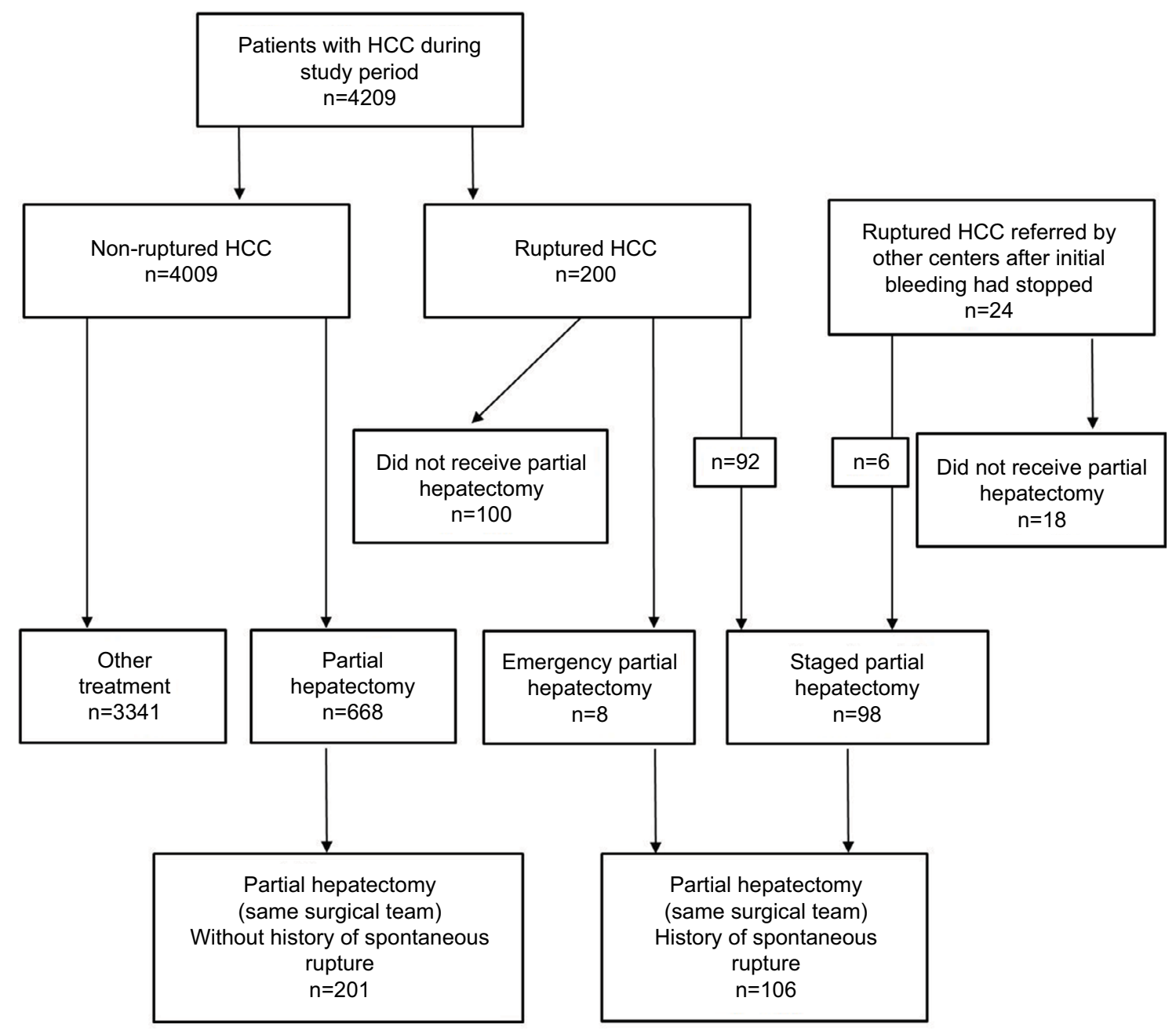

Figure I Study flow chart.

Abbreviation: HCC, hepatocellular carcinoma.

between the two groups. Patients with ruptured HCC were more likely to present with more intraoperative blood loss $(P=0.006)$ and intraoperative blood transfusion $(P<0.001)$ but without a longer duration of operation $(P=0.885)$ compared to those in the non-ruptured group. In addition, there was a lower $\mathrm{R} 0$ resection rate in the ruptured group $(83.9 \%$ vs $87.6 \%$ ). Patients with ruptured HCC were more likely to have a nonanatomical partial hepatectomy. No significant difference in morbidity and mortality was found between both groups (Table 2). One hospital death (due to hepatic failure) occurred in the ruptured group.

\section{Factors associated with spontaneous rupture of $\mathrm{HCC}$}

Univariate analysis indicated that sudden-abdominal pain, underlying diseases of hypertension, liver cirrhosis, ChildPugh grade, hemoglobin, TB, serum albumin level, AFP level, HBsAg, tumor size and ascites were significantly associated with STR (Table 1). That is, STR was more frequently observed in patients with sudden-abdominal pain, hypertension and cirrhosis, positive HBsAg, larger tumor size and a poorer liver functional reserve. However, STR was also more frequently observed in patients with the presence of ascites, a lower serum albumin level, a higher TB and AFP level.

\section{Parameters associated with spontaneous rupture of HCC (multivariate analyses)}

Multivariate analysis with logistic regression revealed the following parameters as independent prognostic factors for STR: maximum tumor diameter (hazard ratio [HR]: 0.187 [ $>5 \mathrm{~cm}$ vs $\leq 5 \mathrm{~cm}]$ ), the presence of hypertension (HR: 0.036), liver cirrhosis (HR: 0.195), TB (HR: 0.373) and the presence of ascites (HR: 0.127; Table 3). Cox proportional hazards analyses of OS and RFS in 307 patients with HCC are shown in Tables 4 and 5. 
Table I Demographic and laboratory data for patients with ruptured and non-ruptured HCC undergoing hepatectomy

\begin{tabular}{|c|c|c|c|}
\hline Variables & Ruptured HCC $(n=106)$ & Non-ruptured HCC $(n=201)$ & $P$-value \\
\hline Age (years) & $47.9(22-75)$ & $50.5(21-78)$ & 0.066 \\
\hline $\operatorname{Sex}(M: F)$ & $99 / 7$ & $179 / 22$ & 0.216 \\
\hline Diabetes & $3 / 103$ & $5 / 196$ & 0.858 \\
\hline Hypertension & $10 / 96$ & $6 / 195$ & $0.016 *$ \\
\hline Liver cirrhosis & $89 / 17$ & $113 / 88$ & $<0.00 I^{*}$ \\
\hline Child-Pugh (A:B) & $96 / 10$ & $199 / 2$ & $<0.00 I^{*}$ \\
\hline PT (s) & $13.0(10.4-\mid 7.8)$ & $13.3(9.8-23.5)$ & 0.165 \\
\hline Hemoglobin (g/L) & $126(60-|4|)$ & $140(90-155)$ & $<0.00$ I* \\
\hline TB $(\mu \mathrm{mol} / \mathrm{L})$ & $21.8(6.3-130)$ & I6.I (5.2-48.3) & $<0.00 I^{*}$ \\
\hline Albumin $(g / L)$ & $39.7(23.2-49.4)$ & $40.8(24.7-55.5)$ & $0.029 *$ \\
\hline ALT (IU/L) & $60.3(9.1-559.8)$ & $59.4(9.6-4 \mid 1.4)$ & 0.892 \\
\hline AST (IU/L) & $67.9(12.4-785.1)$ & $59.9(17.3-353.5)$ & 0.280 \\
\hline Creatinine $(\mu \mathrm{mol} / \mathrm{L})$ & $71.9(39-103)$ & $74.4(35-242)$ & 0.223 \\
\hline $\operatorname{AFP}(\mu g / L)$ & $643.3(1.5-1000)$ & $771.7(2-63159)$ & 0.768 \\
\hline AFP $>100 \mu g / L$ & $75 / 31$ & $113 / 88$ & $0.013^{*}$ \\
\hline Positive $\mathrm{HBsAg}$ & $98 / 8$ & $164 / 37$ & $0.013^{*}$ \\
\hline Tumor size $(\mathrm{cm})$ & $8.6(1.8-17.5)$ & $7.1(I-22)$ & $0.002 *$ \\
\hline Tumor size $>5 \mathrm{~cm}$ & $89 / 17$ & $116 / 85$ & $<0.00 I^{*}$ \\
\hline Tumor location (R/L/both) & $72 / 24 / 10$ & $145 / 38 / 18$ & 0.715 \\
\hline Protrusion & $55 / 51$ & $90 / 111$ & 0.235 \\
\hline Vascular thrombus & $61 / 45$ & $12 \mid / 80$ & 0.653 \\
\hline Capsule formation & $64 / 42$ & $|30 / 7|$ & 0.459 \\
\hline Satellite lesions & $23 / 83$ & $35 / 166$ & 0.362 \\
\hline Ascites & $51 / 55$ & $16 / 185$ & $<0.00 I^{*}$ \\
\hline
\end{tabular}

Notes: Data presented as ratio or mean value (range minimum to maximum). $* P<0.05$.

Abbreviations: AFP, $\alpha$-fetoprotein; ALT, alanine aminotransferase; AST, aspartate aminotransferase; $F$, female; $\mathrm{HBs} A g$, hepatitis B surface antigen; HCC, hepatocellular carcinoma; L, left; M, male; PT, prothrombin time; R, right; TB, total bilirubin.

Table 2 Operative detail of patients with ruptured and non-ruptured HCC undergoing hepatectomy

\begin{tabular}{|c|c|c|c|}
\hline Detail & Ruptured HCC $(n=106)$ & Non-ruptured HCC $(n=20 I)$ & $P$-value \\
\hline Extent of hepatectomy & & & 0.169 \\
\hline Minor & 44 & 100 & \\
\hline Major & 62 & 101 & \\
\hline Type of hepatectomy & & & $0.022 *$ \\
\hline Anatomical & 56 & 133 & \\
\hline Nonanatomical & 50 & 68 & \\
\hline Operating time (min) & $166.8 \pm 70.2$ & $165.6 \pm 69.0$ & 0.885 \\
\hline Duration of clamping (min) & $17.1 \pm 8.5$ & $16.0 \pm 7.3$ & 0.233 \\
\hline Blood loss (mL) & $819.8 \pm 987.0$ & $521.3 \pm 779.5$ & $0.006 *$ \\
\hline Blood transfusion $(\mathrm{mL})$ & $876.1 \pm 1237.8$ & $371.4 \pm 1048.5$ & $<0.00 I^{*}$ \\
\hline Margin $>1 \mathrm{~cm}$ & $7 / 99$ & $26 / 175$ & 0.089 \\
\hline Surgical margins & & & 0.655 \\
\hline RO resection & 89 & 176 & \\
\hline RI resection & 16 & 24 & \\
\hline $\mathrm{R} 2$ resection & 1 & 1 & \\
\hline Hospital death & I & I & 0.644 \\
\hline \multicolumn{4}{|l|}{ Major complications } \\
\hline Postoperative bleeding & 0 & I & 0.467 \\
\hline Liver failure & I & 2 & 0.239 \\
\hline Bile leak & I & 3 & 0.687 \\
\hline Pleural effusion & 51 & 106 & $0.44 I$ \\
\hline Postoperative hospital stay & $20.2 \pm 7.3$ & $18.6 \pm 7.4$ & 0.083 \\
\hline Recurrence & $57 / 49$ & $124 / 77$ & 0.183 \\
\hline
\end{tabular}

Notes: Data presented as number or mean \pm SD. $* P<0.05$.

\section{Long-term survival outcomes}

The duration of follow-up ranged from 1 to 104 months (median 35.9 months). Among the 307 surviving patients,
106 patients were in the ruptured group and 201 patients were in the non-ruptured group. The 1-, 3- and 5-year OS rates for all 307 patients were $54.0 \%, 37.3 \%$ and $33.8 \%$, respectively, 
Table 3 Multiple forward stepwise logistic regression analysis of clinicopathological features

\begin{tabular}{|c|c|c|c|c|c|}
\hline Variables & SE & Wald & HR & 95\% Cl for HR & P-value \\
\hline Hypertension (presence vs absence) & 0.817 & $16.45 \mid$ & 0.036 & $0.01-0.18$ & $<0.001^{*}$ \\
\hline Liver cirrhosis (presence vs absence) & 0.384 & 18.142 & 0.195 & $0.09-0.41$ & $<0.00 I^{*}$ \\
\hline Tumor size $(\leq 5$ vs $>5 \mathrm{~cm})$ & 0.380 & 19.516 & 0.187 & $0.09-0.39$ & $<0.00 I^{*}$ \\
\hline $\mathrm{TB}(\leq 18.8 \mathrm{vs}>18.8 \mu \mathrm{mol} / \mathrm{L})$ & 0.314 & 9.866 & 0.373 & $0.20-0.69$ & $0.002 *$ \\
\hline Ascites (presence vs absence) & 0.393 & 27.499 & 0.127 & $0.06-0.28$ & $<0.00 I^{*}$ \\
\hline
\end{tabular}

Note: $* P<0.05$.

Abbreviations: $\mathrm{Cl}$, confidence interval; $\mathrm{HR}$, hazard ratio; $\mathrm{SE}$, standard error; $\mathrm{TB}$, total bilirubin.

Table 4 Cox proportional hazards analyses of OS in 307 patients with HCC

\begin{tabular}{|c|c|c|c|c|c|}
\hline Variables & SE & Wald & $\operatorname{Exp}(B)$ & $95 \% \mathrm{Cl}$ & P-value \\
\hline Maximum tumor size ( $\geq 5$ vs $<5 \mathrm{~cm})$ & 0.183 & 9.047 & 1.736 & $1.212-2.486$ & 0.003 \\
\hline $\operatorname{AFP}(\geq 400$ vs $<400 \mu g / L)$ & 0.153 & 9.251 & 1.591 & $1.179-2.145$ & 0.002 \\
\hline Tumor type (unifocal vs multiple/diffuse) & 0.169 & 9.704 & 1.692 & $1.215-2.355$ & 0.002 \\
\hline Microscopic vascular invasion (yes vs no) & 0.208 & 16.134 & 2.302 & $1.533-3.459$ & $<0.001$ \\
\hline Child-Pugh grade (A vs B) & 0.417 & 4.068 & 0.431 & $0.190-0.977$ & 0.044 \\
\hline STR (yes vs no) & 0.181 & 12.328 & 1.889 & I.324-2.694 & $<0.001$ \\
\hline
\end{tabular}

Abbreviations: AFP, $\alpha$-fetoprotein; $\mathrm{Cl}$, confidence interval; HCC, hepatocellular carcinoma; OS, overall survival; SE, standard error; STR, spontaneous tumor rupture.

Table 5 Cox proportional hazards analyses of RFS in 307 patients with HCC

\begin{tabular}{llllll}
\hline Variables & SE & Wald & Exp(B) & 95\% Cl & P-value \\
\hline Maximum tumor size $(\geq 5$ vs $<5 \mathrm{~cm})$ & 0.198 & 12.022 & 1.985 & $1.347-2.926$ & 0.001 \\
AFP $(\geq 400$ vs $<400 \mu \mathrm{g} / \mathrm{L})$ & 0.167 & 4.927 & 1.448 & $1.044-2.009$ & 0.026 \\
Microscopic vascular invasion (yes vs no) & 0.225 & 9.622 & 2.009 & $1.293-3.121$ & 0.002 \\
STR (yes vs no) & 0.203 & 0.077 & 0.945 & $0.635-1.407$ & 0.782 \\
\hline
\end{tabular}

Abbreviations: AFP, $\alpha$-fetoprotein; $\mathrm{Cl}$, confidence interval; HCC, hepatocellular carcinoma; RFS, recurrence-free survival; SE, standard error; STR, spontaneous tumor rupture.

with a median OS of 17 months $(95 \%$ confidence interval [CI]: 12.0-25.0). The 1-, 3- and 5-year OS rates for the 307 survivors who had an R0 resection were $88.8 \%, 64.6 \%$ and $53.7 \%$, respectively, with a median OS of 41 months. The Kaplan-Meier survival curves of RFS and overall actuarial survival of both groups of patients are shown in Figure 2. The median disease-free survival was 10 months $(95 \% \mathrm{CI}$ : 5.0-16.0) in the ruptured HCC group and 23 months $(95 \%$ CI: 14.0-32.0) in the non-ruptured HCC group.

After partial hepatectomy, 92 (86.8\%) of 106 patients in the ruptured group and $124(61.7 \%)$ of 201 patients in the non-ruptured group died during follow-up. Peritoneal dissemination developed in 20 (7.5\%) of 265 patients after R0 resection and occurred more often in the ruptured group: 29 (32.6\%) of 89 patients versus nine $(5.1 \%)$ of 176 patients $(P<0.001)$.

\section{Impact of STR on survival}

The ruptured HCC group had significantly lower OS rates (37.7\%, 19.7\% and $14.7 \%$ at 1,3 and 5 years, respectively) than the non-ruptured HCC group $(77.7 \%, 56.9 \%$ and $41.6 \%$; $P<0.001$; Figure 2A). There was also a significant difference in RFS between the ruptured $(45.8 \%, 34.2 \%$ and $31.3 \%$ at 1 , 3 and 5 years, respectively) and non-ruptured (58.2\%, 39.7\% and $36.0 \%$ ) groups ( $P=0.016$; Figure $2 \mathrm{~B}$ ).

Both univariable and multivariable analyses showed that STR was independently associated with poor OS (HR 1.89, 95\% CI: 1.32-2.69). Furthermore, although univariable analyses showed that RFS was lower in the ruptured group than in the non-ruptured group $(P=0.016)$, STR could not independently predict poor RFS after partial hepatectomy for HCC in multivariable analyses (HR 0.95, 95\% CI: 0.64-1.41, $P=0.782$; Tables S1 and S2).

\section{Discussion}

The incidence of spontaneous rupture of HCC shows an obvious geographical difference in previously reported studies, and $\sim 10 \%-15 \%$ of patients with HCC develop this complication. ${ }^{7}$ In the present study, 106 (13.7\%) of the 774 patients underwent hepatic resection due to HCC rupture.

In this study, we found a similar baseline characteristic between groups with and without rupture in physical findings, biochemical data, hepatitis status and extent of cirrhosis. Patients with ruptured HCC were more likely to have clinical 
A

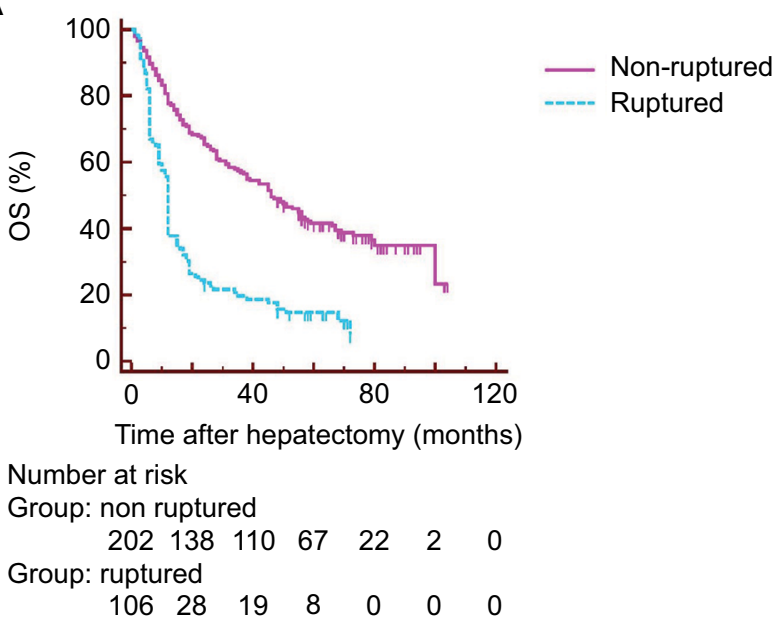

B

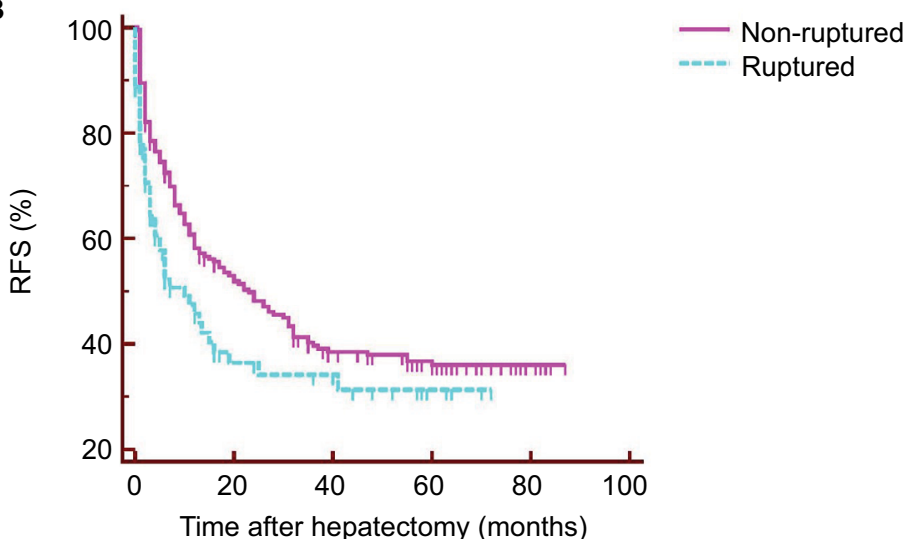

Number at risk

Group: non ruptured

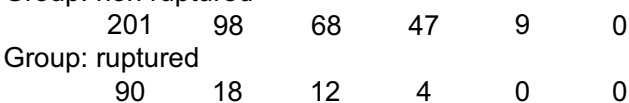

Figure 2 (A) OS and (B) RFS after hepatectomy for ruptured and non-ruptured HCC.

Note: (A and B) $P<0.001$ (log-rank test).

Abbreviations: $\mathrm{HCC}$, hepatocellular carcinoma; OS, overall survival; RFS, recurrence-free survival.

manifestations such as sudden attack of severe abdominal pain, signs of bleeding during physical examination, a lower hemoglobin level, a larger tumor size, higher TB levels and greater intraoperative blood loss during hepatic resection.

Sudden abdominal pain in hemodynamic unstable HCC patients should indicate the diagnosis of ruptured HCC. Nevertheless, multiple logistic regression analysis did not confirm sudden-onset abdominal pain to be a prognostic factor in patients with ruptured HCC, which was not consistent with the study by Yeh et al. ${ }^{10} \mathrm{~A}$ possible explanation may be that the patients enrolled in the present series were all treated in a single surgical team. Moreover, we found that STR was more frequent among patients with a larger tumor size and a poorer liver functional reserve (a higher serum TB level and presence of ascites), which is consistent with previous reports. ${ }^{6}, 15$

Interestingly, we also found the presence of hypertension and liver cirrhosis to be predictive factors for spontaneous rupture of HCC. It may be attributed to the fact that hypertension can directly result in an increase in pressure within the tumor; the rupture of a vascular tumor like HCC may thus lead to the tearing of vessels with uncontrollable blood loss. The condition may be further exacerbated in cirrhotic patients with underlying coagulopathy. Both of the two factors may promote the process of rupture described earlier. ${ }^{16}$

Treatments for ruptured HCC include control of hemorrhage and liver resection when possible. The feasibility of hepatectomy depends largely on the extent of the tumor 
(e.g., caval involvement). Hepatic resection is technically challenging in cirrhotic livers. Lai et al ${ }^{17}$ reported that $60.7 \%$ of patients with ruptured HCC had macroscopic cirrhosis, and only $12.5 \%$ of patients were managed by hepatectomy. Dewar et a $1^{18}$ reported that 36 of 37 patients with ruptured HCC had underlying cirrhosis of the liver, 11 of whom underwent hepatectomy. In our study, 89 of the 106 patients with ruptured $\mathrm{HCC}$ had underlying cirrhosis, and over half of the patients $(60.4 \%, 64 / 106)$ underwent resection. With recent improvements in hepatic surgery, hepatectomy for ruptured HCC is feasible and may be potentially curative.

Previous Japanese ${ }^{19}$ and Italian ${ }^{20}$ studies have confirmed that emergency hepatic resection for ruptured HCC may achieve a long-term outcome comparable with that of elective surgery in selected patients. We also demonstrated that patients with ruptured $\mathrm{HCC}$ who underwent hepatectomy had worse OS compared with those in the non-ruptured HCC group.

Although it is generally presumed that STR is a risk factor impacting the prognosis of HCC patients undergoing hepatic resection, controversies remain on this issue. ${ }^{9,10,21}$ For instance, Aoki et $\mathrm{al}^{6}$ and Liu et $\mathrm{al}^{9}$ reported that the survival of patients with ruptured HCC was significantly worse than that of patients with non-ruptured HCC. In contrast, Yeh et a ${ }^{10}$ reported that patients with ruptured HCC had a similar OS compared to those with non-ruptured HCC; however, disease-free survival rate was significantly lower in those with ruptured HCC. As observed elsewhere, ${ }^{19}$ a trend toward lower disease-free survival rate was observed in patients with tumor rupture who underwent hepatic resection. The present study has identified in a large patient cohort that tumor rupture itself had a negative impact on patient survival; furthermore, of note, STR, which was not independently associated with poorer RFS (HR 0.95, 0.64-1.41) after hepatectomy, predicted poor OS and RFS after hepatectomy for HCC.

It is worth pointing out that all surgeons in our unit had abundant operating experience of hepatectomies. As a result, the rate of hepatectomy in the present cohort was much higher than previous counterparts. ${ }^{9,20}$ The 1-, 3- and 5-year OS and RFS rates after hepatectomy for ruptured HCC were comparable with those reported elsewhere. ${ }^{9,10,22}$

Perioperative morbidity and mortality rates were comparable between both groups, suggesting that hepatectomy did not pose additional perioperative risk for ruptured HCC in selected patients whose operations were performed by experienced surgeons. Peritoneal dissemination is not uncommon after curative resection of ruptured HCC, ${ }^{22}$ DWPL and intraperitoneal 5-FU were thus administered to prevent peritoneal dissemination. DWPL would remove tumor cells to a large extent, and thus, tumor recurrence would be delayed and better survival would be achieved in patients with spontaneously ruptured HCCs. ${ }^{14,23}$ Currently, the role of 5-FU in HCC treatment regarding adjuvant therapy after surgery, based on a randomized, controlled trial, placebo controlled, ${ }^{24}$ that showed lower recurrence rate and higher time-to-tumor progression as well as 5-year OS was better in the 5-FU group. In addition to the tumors being more advanced in the ruptured group, the R0 resection rate was significantly lower than that in the non-ruptured group (83.9\% vs 87.6 $\%$ ). In some patients with ruptured HCC, the intraoperative extent of tumor was more advanced than on preoperative assessment, with intraperitoneal seeding being detected at operation. Finally, peritoneal secondaries were more often found on follow-up in the ruptured group after R0 resection.

Yamagata et a $\mathrm{l}^{19}$ claimed that increased intratumor pressure with venous invasion was the main culprit for recurrence in the ruptured HCC. However, in the present series, a similar percentage of vascular invasion was observed between ruptured and non-ruptured groups. Greater blood loss and more blood transfusion needs might contribute to the poorer disease-free survival in patients with ruptured HCC. Perioperative blood transfusion has been related to shorter disease-free survival because of the immunosuppressive effect of transfusion..$^{25}$

Battula et $\mathrm{al}^{26}$ reported that the multifocality of the tumor and large tumor size can help predict the risk of decompensation and poor outcomes. Kirikoshi et $\mathrm{al}^{27}$ demonstrated that tumor size was the only independent factor for long-term survival among patients who underwent successful initial TAE. In the present series, tumor size was an important factor influencing survival in patients undergoing successful hepatectomy for ruptured HCC; moreover, we identified that tumor size was an independent factor influencing OS (HR: 1.74, 95\% CI: $1.21-2.49, P=0.003$ ) and RFS (HR: $1.99,95 \%$ CI: $1.35-2.93, P=0.001)$.

In addition, the present study, together with the studies of Sun et $\mathrm{a}^{28}$ and Hung et al, ${ }^{29}$ showed that hepatitis B positivity was an independent factor for OS and disease-free survival and was associated with a higher risk of early recurrence and poorer survival in patients after curative resection of hepatitis B virus (HBV)-related HCC (within 1 year), ${ }^{28-30}$ suggesting HBsAg positivity impact on OS and RFS after partial hepatectomy for HCC. Although there was not a significant difference in OS or RFS between the ruptured 
and non-ruptured groups, the HBsAg-negative group had lower OS rates $(63.2 \%, 45.6 \%$ and $42.1 \%$ at 1,3 and 5 years respectively) than the HBsAg-positive group (52.1\%, 35.4\%, $32 \%$ ); meanwhile, the 1-, 3-, 5-year OS rates for HBsAgnegative patients were $70.5 \%, 54.4 \%$ and $43.1 \%$, respectively, in contrast to $62.7 \%, 42.2 \%$ and $30.2 \%$ for HBsAg-positive patients.

The present study has several flaws. First, although the data were collected prospectively, this is actually a retrospective cohort study with all its inherent shortcomings. Second, the study population in the present study was limited to Chinese HCC patients and the dominant etiology of the liver disease was HBV, which is different from that seen in Western countries.

\section{Conclusion}

The presence of hypertension, liver cirrhosis, higher serum TB level, tumor size $>5 \mathrm{~cm}$ and ascites are the independent indicators of ruptured HCC. The present study confirmed that tumor rupture itself had a negative impact on patient survival, and hepatic resection for patients with spontaneous $\mathrm{HCC}$ rupture is often feasible and is the treatment of choice for ruptured $\mathrm{HCC}$, which can result in OS and RFS rates that are comparable to those of patients without rupture.

\section{Acknowledgments}

We acknowledge Jing Zhao and Ye Cai for professional statistical analysis. This work was supported by grants from the Natural Science Foundation of Hubei Province (2016CFB442 and WJ2017M236). This study was registered on November 30, 2014 under clinical trial number ChiCTROCS-11001300. Please contact author for data requests.

\section{Author contributions}

All authors contributed toward data analysis, drafting and critically revising the paper and agree to be accountable for all aspects of the work.

\section{Disclosure}

The authors report no conflicts of interest in this work.

\section{References}

1. Chen W, Zheng R, Baade PD, et al. Cancer statistics in China, 2015. CA Cancer J Clin. 2016;66(2):115-132.

2. Torre LA, Bray F, Siegel RL, Ferlay J, Lortet-Tieulent J, Jemal A. Global cancer statistics, 2012. CA Cancer J Clin. 2015;65(2):87-108.

3. Llovet JM, Burroughs A, Bruix J. Hepatocellular carcinoma. Lancet. 2003;362(9399):1907-1917.

4. Yang T, Sun YF, Zhang J, et al. Partial hepatectomy for ruptured hepatocellular carcinoma. Br J Surg. 2013;100(8):1071-1079.
5. Yoshida H, Mamada Y, Taniai N, Uchida E. Spontaneous ruptured hepatocellular carcinoma. Hepatol Res. 2016;46(1):13-21.

6. Aoki T, Kokudo N, Matsuyama Y, et al; Liver Cancer Study Group of Japan. Prognostic impact of spontaneous tumor rupture in patients with hepatocellular carcinoma: an analysis of 1160 cases from a nationwide survey. Ann Surg. 2014;259(3):532-542.

7. Lai EC, Lau WY. Spontaneous rupture of hepatocellular carcinoma: a systematic review. Arch Surg. 2006;141(2):191-198.

8. Leung KL, Lau WY, Lai PB, Yiu RY, Meng WC, Leow CK. Spontaneous rupture of hepatocellular carcinoma: conservative management and selective intervention. Arch Surg. 1999;134(10):1103-1107.

9. Liu CL, Fan ST, Lo CM, et al. Management of spontaneous rupture of hepatocellular carcinoma: single-center experience. J Clin Oncol. 2001;19(17):3725-3732.

10. Yeh CN, Lee WC, Jeng LB, Chen MF, Yu MC. Spontaneous tumour rupture and prognosis in patients with hepatocellular carcinoma. $\mathrm{Br} J$ Surg. 2002;89(9):1125-1129.

11. Yang T, Zhang J, Lu JH, et al. A new staging system for resectable hepatocellular carcinoma: comparison with six existing staging systems in a large Chinese cohort. J Cancer Res Clin Oncol. 2011;137(5):739-750.

12. Pugh RN, Murray-Lyon IM, Dawson JL, Pietroni MC, Williams R. Transection of the oesophagus for bleeding oesophageal varices. $\mathrm{Br} J$ Surg. 1973;60(8):646-649.

13. Xia F, Lau WY, Qian C, Ma K, Li X, Bie P. Continuous occlusion of hepatic artery proper for prevention of blood loss in partial hepatectomy for ruptured hepatocellular carcinoma: a case-matched comparative study. Ann Surg Oncol. 2011;18(6):1638-1643.

14. Lin CH, Hsieh HF, Yu JC, Chen TW, Yu CY, Hsieh CB. Peritoneal lavage with distilled water during liver resection in patients with spontaneously ruptured hepatocellular carcinomas. J Surg Oncol. 2006;94(3):255-256.

15. Tanaka A, Takeda R, Mukaihara S, et al. Treatment of ruptured hepatocellular carcinoma. Int J Clin Oncol. 2001;6(6):291-295.

16. Zhu Q, Li J, Yan JJ, Huang L, Wu MC, Yan YQ. Predictors and clinical outcomes for spontaneous rupture of hepatocellular carcinoma. World J Gastroenterol. 2012;18(48):7302-7307.

17. Lai EC, Wu KM, Choi TK, Fan ST, Wong J. Spontaneous ruptured hepatocellular carcinoma. An appraisal of surgical treatment. Ann Surg. 1989;210(1):24-28.

18. Dewar GA, Griffin SM, Ku KW, Lau WY, Li AK. Management of bleeding liver tumours in Hong Kong. Br J Surg. 1991;78(4): 463-466.

19. Yamagata M, Maeda T, Ikeda Y, Shirabe K, Nishizaki T, Koyanagi N. Surgical results of spontaneously ruptured hepatocellular carcinoma. Hepatogastroenterology. 1995;42(5):461-464.

20. Vergara V, Muratore A, Bouzari H, et al. Spontaneous rupture of hepatocelluar carcinoma: surgical resection and long-term survival. Eur $J$ Surg Oncol. 2000;26(8):770-772.

21. Mizuno S, Yamagiwa K, Ogawa T, et al. Are the results of surgical treatment of hepatocellular carcinoma poor if the tumor has spontaneously ruptured? Scand J Gastroenterol. 2004;39(6):567-570.

22. Shuto T, Hirohashi K, Kubo S, et al. Delayed hepatic resection for ruptured hepatocellular carcinoma. Surgery. 1998;124(1):33-37.

23. Brundell SM, Tucker K, Chatterton B, Hewett PJ. The effect of lavage on intraabdominal cell burden. Surg Endosc. 2002;16(7): 1064-1067.

24. Xia Y, Qiu Y, Li J, et al. Adjuvant therapy with capecitabine postpones recurrence of hepatocellular carcinoma after curative resection: a randomized controlled trial. Ann Surg Oncol. 2010;17(12):3137-3144.

25. Tung-Ping Poon R, Fan ST, Wong J. Risk factors, prevention, and management of postoperative recurrence after resection of hepatocellular carcinoma. Ann Surg. 2000;232(1):10-24.

26. Battula N, Madanur M, Priest O, et al. Spontaneous rupture of hepatocellular carcinoma: a Western experience. Am J Surg. 2009;197(2): 164-167.

27. Kirikoshi H, Saito S, Yoneda M, et al. Outcomes and factors influencing survival in cirrhotic cases with spontaneous rupture of hepatocellular carcinoma: a multicenter study. BMC Gastroenterol. 2009;9:29. 
28. Sun HC, Zhang W, Qin LX, et al. Positive serum hepatitis B e antigen is associated with higher risk of early recurrence and poorer survival in patients after curative resection of hepatitis B-related hepatocellular carcinoma. J Hepatol. 2007;47(5):684-690.

29. Hung IF, Poon RT, Lai CL, Fung J, Fan ST, Yuen MF. Recurrence of hepatitis B-related hepatocellular carcinoma is associated with high viral load at the time of resection. Am J Gastroenterol. 2008;103(7):1663-1673.
30. Shah SA, Cleary SP, Wei AC, et al. Recurrence after liver resection for hepatocellular carcinoma: risk factors, treatment, and outcomes. Surgery. 2007;141(3):330-339. 


\section{Supplementary materials}

Table SI Univariable and multivariable analyses of overall survival in 307 patients with hepatocellular carcinoma

\begin{tabular}{|c|c|c|c|c|c|c|c|}
\hline \multirow[t]{2}{*}{ Variable } & \multirow[t]{2}{*}{$\mathbf{n}$} & \multicolumn{3}{|c|}{ Overall survival (\%) } & \multirow[t]{2}{*}{ Univariable $\boldsymbol{P}^{\sharp}$} & \multirow[t]{2}{*}{ Multivariable $P^{*}$} & \multirow[t]{2}{*}{ Hazard ratio } \\
\hline & & I year & 3 years & 5 years & & & \\
\hline \multicolumn{8}{|l|}{ Sex } \\
\hline Male & 278 & 63.4 & 43.3 & 31.7 & 0.403 & & \\
\hline Female & 29 & 69.0 & 51.7 & 37.9 & & & \\
\hline \multicolumn{8}{|l|}{ Age (years) } \\
\hline$<60$ & 255 & 62.4 & 42.3 & 33.8 & 0.929 & & \\
\hline$\geq 60$ & 52 & 71.7 & 52.8 & 25.0 & & & \\
\hline \multicolumn{8}{|l|}{ Presentation } \\
\hline Subclinical & 149 & 87.9 & 66.0 & 54.9 & 0.016 & 0.184 & \\
\hline Symptomatic & 158 & 79.2 & 48.5 & 39.7 & & & \\
\hline \multicolumn{8}{|l|}{$\mathrm{HBsAg}$} \\
\hline Positive & 263 & 62.7 & 42.2 & 30.2 & 0.062 & & \\
\hline Negative & 44 & 70.5 & 54.4 & 43.1 & & & \\
\hline \multicolumn{8}{|l|}{$\mathrm{TB}(\mu \mathrm{mol} / \mathrm{L})$} \\
\hline$<18.8$ & 192 & 68.2 & 49.0 & 37.7 & 0.007 & $0.87 I$ & \\
\hline$\geq 18.8$ & 115 & 56.9 & 36.0 & 23.0 & & & \\
\hline \multicolumn{8}{|c|}{$\alpha$-Fetoprotein (mg/L) } \\
\hline$<400$ & 146 & 80.8 & 58.8 & 43.9 & $<0.001$ & 0.002 & $1.59(1.18,2.15)$ \\
\hline$\geq 400$ & 161 & 48.8 & 30.9 & 22.0 & & & \\
\hline \multicolumn{8}{|l|}{ PT (s) } \\
\hline$<13$ & 149 & 63.1 & 40.3 & 31.8 & 0.938 & & \\
\hline$\geq 13$ & 158 & 64.8 & 47.7 & 32.7 & & & \\
\hline \multicolumn{8}{|l|}{ Child-Pugh grade } \\
\hline$A$ & 295 & 64.9 & 44.5 & 32.3 & $<0.001$ & 0.044 & $0.43(0.19,0.98)$ \\
\hline B & 12 & 41.7 & 33.3 & 27.5 & & & \\
\hline \multicolumn{8}{|l|}{ Cirrhosis } \\
\hline Yes & 105 & 67.6 & 48.6 & 34.9 & 0.385 & & \\
\hline No & 202 & 62.1 & 41.8 & 31.0 & & & \\
\hline \multicolumn{8}{|l|}{ ALT (units/L) } \\
\hline$<40$ & 133 & 60.9 & 47.3 & 35.4 & 0.387 & & \\
\hline$\geq 40$ & 174 & 66.3 & 41.7 & 30.0 & & & \\
\hline \multicolumn{8}{|l|}{ AST (units/L) } \\
\hline$<40$ & 103 & 73.8 & 56.2 & 44.8 & 0.008 & 0.326 & \\
\hline$\geq 40$ & 204 & 59.0 & 38.0 & 26.0 & & & \\
\hline \multicolumn{8}{|c|}{ Spontaneous tumor rupture } \\
\hline Yes & 106 & 37.7 & 19.7 & 14.7 & $<0.001$ & $<0.001$ & $1.89(1.32,2.69)$ \\
\hline No & 201 & 77.7 & 56.9 & 41.6 & & & \\
\hline \multicolumn{8}{|c|}{ Maximum tumor size $(\mathrm{cm})$} \\
\hline$<5$ & 103 & 88.3 & 67.9 & 52.9 & $<0.001$ & 0.003 & $1.74(\mathrm{I} .2 \mathrm{I}, 2.49)$ \\
\hline$\geq 5$ & 204 & 51.7 & 32.2 & 21.9 & & & \\
\hline Macroscopic vas & & & & & & & \\
\hline Yes & 125 & 45.2 & 12.9 & 5.7 & $<0.001$ & 0.806 & \\
\hline No & 182 & 64.5 & 45.8 & 35.7 & & & \\
\hline Microscopic vasc & & & & & & & \\
\hline Yes & 272 & 27.8 & II.I & 8.3 & $<0.001$ & $<0.001$ & $2.30(1.53,3.46)$ \\
\hline No & 35 & 68.7 & 48.5 & 35.5 & & & \\
\hline Extrahepatic spr & & & & & & & \\
\hline Yes & 67 & 41.2 & 26.5 & 20.6 & $<0.001$ & 0.155 & \\
\hline No & 240 & 70.4 & 49.1 & 35.7 & & & \\
\hline Tumor differenti & & & & & & & \\
\hline I-II & 45 & 92.5 & 77.0 & 66.9 & $<0.001$ & 0.080 & \\
\hline III-IV & 262 & 79.7 & 53.1 & 43.3 & & & \\
\hline
\end{tabular}


Table SI (Continued)

\begin{tabular}{|c|c|c|c|c|c|c|c|}
\hline & \multirow[t]{2}{*}{$\mathbf{n}$} & \multicolumn{3}{|c|}{ Overall survival (\%) } & \multirow[t]{2}{*}{ Univariable $\boldsymbol{P}^{\sharp}$} & \multirow[t]{2}{*}{ Multivariable $P^{*}$} & \multirow[t]{2}{*}{ Hazard ratio } \\
\hline & & I year & 3 years & 5 years & & & \\
\hline \multicolumn{8}{|l|}{ Tumor type } \\
\hline Unifocal & 242 & 70.2 & 50.4 & 36.6 & $<0.001$ & 0.002 & $1.69(1.22,2.36)$ \\
\hline Multiple/diffuse & 65 & 40.9 & 21.2 & 16.4 & & & \\
\hline \multicolumn{8}{|l|}{ Blood loss (mL) } \\
\hline$<500$ & 200 & 86.5 & 65.9 & 56.2 & 0.004 & 0.084 & \\
\hline$\geq 500$ & 107 & 72.0 & 40.0 & 29.0 & & & \\
\hline \multicolumn{8}{|l|}{ Blood transfusion } \\
\hline Yes & 83 & 66.7 & 34.0 & 23.2 & $<0.001$ & 0.221 & \\
\hline No & 224 & 87.8 & 65.6 & 55.9 & & & \\
\hline \multicolumn{8}{|c|}{ Type of hepatectomy } \\
\hline Anatomical & 189 & 82.4 & 57.5 & 47.0 & 0.600 & & \\
\hline Non-anatomical & 118 & 81.1 & 54.9 & 46.0 & & & \\
\hline \multicolumn{8}{|c|}{ Extent of hepatectomy } \\
\hline Major & 163 & 80.0 & 54.8 & 44.2 & 0.130 & & \\
\hline Minor & 144 & 85.1 & 60.0 & 47.3 & & & \\
\hline \multicolumn{8}{|c|}{ Surgical resection margin } \\
\hline RO & 265 & 88.8 & 64.6 & 53.7 & $<0.001$ & 0.057 & \\
\hline RI & 40 & 43.8 & 9.8 & 5.8 & & & \\
\hline $\mathrm{R} 2$ & 2 & 18.9 & 4.9 & 2.0 & & & \\
\hline
\end{tabular}

Notes: Values in parentheses are $95 \%$ confidence intervals. " Log rank test. $*$ Variables with univariable $P<0.05$ were entered in the Cox regression model.

Abbreviations: ALT, alanine aminotransferase; AST, aspartate aminotransferase; HBsAg, hepatitis B surface antigen; HCV, hepatitis C virus; PT, prothrombin time; TB, total bilirubin.

Table S2 Univariable and multivariable analyses of recurrence-free survival in 307 patients with hepatocellular carcinoma

\begin{tabular}{|c|c|c|c|c|c|c|c|}
\hline \multirow[t]{2}{*}{ Variable } & \multirow[t]{2}{*}{$\mathbf{n}$} & \multicolumn{3}{|c|}{ Recurrence-free survival (\%) } & \multirow[t]{2}{*}{ Univariable $\boldsymbol{P}^{\sharp}$} & \multirow[t]{2}{*}{ Multivariable $P^{*}$} & \multirow[t]{2}{*}{ Hazard ratio } \\
\hline & & I year & 3 years & 5 years & & & \\
\hline \multicolumn{8}{|l|}{$\overline{\text { Sex }}$} \\
\hline Male & 278 & 53.5 & 36.3 & 33.1 & 0.566 & & \\
\hline Female & 29 & 58.2 & 45.8 & 38.8 & & & \\
\hline \multicolumn{8}{|l|}{ Age (years) } \\
\hline$<60$ & 255 & 52.7 & 37.4 & 34.0 & 0.778 & & \\
\hline$\geq 60$ & 52 & 59.8 & 36.3 & 31.7 & & & \\
\hline \multicolumn{8}{|l|}{ Presentation } \\
\hline Subclinical & 159 & 80.1 & 59.5 & 43.5 & $<0.001$ & 0.095 & \\
\hline Symptomatic & 148 & 65.4 & 44.3 & 29.0 & & & \\
\hline \multicolumn{8}{|l|}{$\mathrm{HBsAg}$} \\
\hline Positive & 263 & 52.1 & 35.4 & 32.0 & 0.139 & & \\
\hline Negative & 44 & 63.2 & 45.6 & 42.1 & & & \\
\hline \multicolumn{8}{|l|}{ TB $(\mu \mathrm{mol} / \mathrm{L})$} \\
\hline$<18.8$ & 192 & 49.1 & 30.3 & 28.6 & 0.157 & & \\
\hline$\geq 18.8$ & 115 & 56.8 & 41.1 & 36.7 & & & \\
\hline \multicolumn{8}{|c|}{$\alpha$-Fetoprotein (mg/L) } \\
\hline$<400$ & 145 & 66.6 & 45.0 & 40.2 & $<0.001$ & 0.026 & $1.45(1.04,2.01)$ \\
\hline$\geq 400$ & 162 & 41.4 & 29.8 & 27.7 & & & \\
\hline \multicolumn{8}{|l|}{ PT (s) } \\
\hline$<13$ & 149 & 56.4 & 39.4 & 36.3 & 0.580 & & \\
\hline$\geq 13$ & 158 & 51.9 & 36.2 & 31.5 & & & \\
\hline \multicolumn{8}{|c|}{ Child-Pugh grade } \\
\hline$A$ & 295 & 54.1 & 37.0 & 33.4 & 0.738 & & \\
\hline B & 12 & 48.6 & 30.2 & 20.2 & & & \\
\hline \multicolumn{8}{|l|}{ Cirrhosis } \\
\hline Yes & 202 & 51.4 & 35.8 & 32.2 & 0.740 & & \\
\hline No & 105 & 58.7 & 38.4 & 35.0 & & & \\
\hline
\end{tabular}


Table S2 (Continued)

\begin{tabular}{|c|c|c|c|c|c|c|c|}
\hline & \multirow[t]{2}{*}{$n$} & \multicolumn{3}{|c|}{ Recurrence-free survival (\%) } & \multirow[t]{2}{*}{ Univariable $\boldsymbol{P}^{\sharp}$} & \multirow[t]{2}{*}{ Multivariable $P^{*}$} & \multirow[t]{2}{*}{ Hazard ratio } \\
\hline & & I year & 3 years & 5 years & & & \\
\hline \multicolumn{8}{|l|}{$\overline{\mathrm{ALT} \text { (units/L) }}$} \\
\hline$<40$ & 133 & 54.9 & 39.5 & 34.2 & 0.899 & & \\
\hline$\geq 40$ & 174 & 52.6 & 35.5 & 33.7 & & & \\
\hline \multicolumn{8}{|l|}{ AST (units/L) } \\
\hline$<40$ & 103 & 65.9 & 46.0 & 40.5 & 0.029 & 0.326 & \\
\hline$\geq 40$ & 204 & 54.3 & 34.8 & 30.5 & & & \\
\hline \multicolumn{8}{|c|}{ Spontaneous tumor rupture } \\
\hline Yes & 106 & 45.8 & 34.2 & 31.3 & 0.016 & 0.782 & $0.95(0.64, \mathrm{I} .4 \mathrm{I})$ \\
\hline No & 201 & 58.2 & 39.7 & 36.0 & & & \\
\hline \multicolumn{8}{|c|}{ Maximum tumor size $(\mathrm{cm})$} \\
\hline$<5$ & 103 & 78.1 & 52.4 & 50.1 & $<0.001$ & 0.001 & $1.99(1.35,2.93)$ \\
\hline$\geq 5$ & 204 & 40.6 & 29.8 & 24.4 & & & \\
\hline \multicolumn{8}{|c|}{ Macroscopic vascular invasion } \\
\hline Yes & 124 & 55.1 & 34.6 & 2 & 0.664 & & \\
\hline No & 183 & 53.1 & 32.9 & 45 & & & \\
\hline \multicolumn{8}{|c|}{ Microscopic vascular invasion } \\
\hline Yes & 270 & 10.4 & 10.1 & 6.9 & $<0.001$ & 0.002 & $2.01(1.29,3.12)$ \\
\hline No & 37 & 59.2 & 40.5 & 37.1 & & & \\
\hline \multicolumn{8}{|l|}{ Extrahepatic spread } \\
\hline Yes & 68 & 35.7 & 27.0 & 19.2 & $<0.001$ & 0.064 & \\
\hline No & 239 & 58.9 & 40.1 & 37.6 & & & \\
\hline \multicolumn{8}{|l|}{ Tumor differentiation } \\
\hline I-II & 50 & 85.0 & 72.0 & 67.0 & $<0.001$ & 0.511 & \\
\hline III-IV & 257 & 66.0 & 44.2 & 35.4 & & & \\
\hline \multicolumn{8}{|l|}{ Tumor type } \\
\hline Unifocal & 242 & 58.8 & 40.9 & 37.0 & $<0.001$ & $0.66 \mathrm{I}$ & \\
\hline Multiple/diffuse & 65 & 35.5 & 23.7 & 21.7 & & & \\
\hline \multicolumn{8}{|c|}{ Perioperative blood loss (mL) } \\
\hline$<500$ & 209 & 75.2 & 56.0 & 46.0 & $<0.001$ & 0.165 & \\
\hline$\geq 500$ & 98 & 58.0 & 34.5 & 28.7 & & & \\
\hline \multicolumn{8}{|c|}{ Intraoperative blood transfusion } \\
\hline Yes & 76 & 53.0 & 30.2 & 22.5 & $<0.001$ & 0.057 & \\
\hline No & 231 & 74.5 & 54.9 & 45.4 & & & \\
\hline \multicolumn{8}{|l|}{ Type of hepatectomy } \\
\hline Anatomical & 189 & 68.8 & 49.4 & 40.2 & 0.899 & & \\
\hline Non-anatomical & 118 & 68.6 & 48.9 & 40.8 & & & \\
\hline \multicolumn{8}{|c|}{ Extent of hepatectomy } \\
\hline Major & 163 & 61.9 & 44.0 & 31.0 & 0.079 & & \\
\hline Minor & 144 & 70.5 & 50.9 & 41.6 & & & \\
\hline
\end{tabular}

Notes: Values in parentheses are $95 \%$ confidence intervals. "Log rank test. *Variables with univariable $P<0.05$ were entered in the Cox regression model.

Abbreviations: ALT, alanine aminotransferase; AST, aspartate aminotransferase; HBsAg, hepatitis B surface antigen; HCV, hepatitis C virus; PT, prothrombin time; TB, total bilirubin.

Cancer Management and Research

\section{Publish your work in this journal}

Cancer Management and Research is an international, peer-reviewed open access journal focusing on cancer research and the optimal use of preventative and integrated treatment interventions to achieve improved outcomes, enhanced survival and quality of life for the cancer patient. The manuscript management system is completely online and includes

\section{Dovepress}

a very quick and fair peer-review system, which is all easy to use. Visit $\mathrm{http}: / / \mathrm{www}$.dovepress.com/testimonials.php to read real quotes from published authors. 